\title{
Internalized Timing of Isochronous Sounds Is Represented in Neuromagnetic Beta Oscillations
}

\author{
Takako Fujioka, ${ }^{1,2}$ Laurel J. Trainor, ${ }^{1,2}$ Edward W. Large, ${ }^{4}$ and Bernhard Ross ${ }^{1,3}$ \\ ${ }^{1}$ Rotman Research Institute, Baycrest Centre, Toronto, Ontario M6A 2E1, Canada, ${ }^{2}$ Department of Psychology, Neuroscience and Behaviour, McMaster \\ University, Hamilton, Ontario L8S 4K1, Canada, ${ }^{3}$ Department of Medical Biophysics, University of Toronto, Toronto, Ontario M5G 2M9, Canada, and \\ ${ }^{4}$ Center for Complex Systems and Brain Sciences, Florida Atlantic University, Boca Raton, Florida 33431
}

\begin{abstract}
Moving in synchrony with an auditory rhythm requires predictive action based on neurodynamic representation of temporal information. Although it is known that a regular auditory rhythm can facilitate rhythmic movement, the neural mechanisms underlying this phenomenon remain poorly understood. In this experiment using human magnetoencephalography, 12 young healthy adults listened passively to an isochronous auditory rhythm without producing rhythmic movement. We hypothesized that the dynamics of neuromagnetic beta-band oscillations $(\sim 20 \mathrm{~Hz})$ - which are known to reflect changes in an active status of sensorimotor functions-would show modulations in both power and phase-coherence related to the rate of the auditory rhythm across both auditory and motor systems. Despite the absence of an intention to move, modulation of beta amplitude as well as changes in cortico-cortical coherence followed the tempo of sound stimulation in auditory cortices and motor-related areas including the sensorimotor cortex, inferior-frontal gyrus, supplementary motor area, and the cerebellum. The time course of beta decrease after stimulus onset was consistent regardless of the rate or regularity of the stimulus, but the time course of the following beta rebound depended on the stimulus rate only in the regular stimulus conditions such that the beta amplitude reached its maximum just before the occurrence of the next sound. Our results suggest that the time course of beta modulation provides a mechanism for maintaining predictive timing, that beta oscillations reflect functional coordination between auditory and motor systems, and that coherence in beta oscillations dynamically configure the sensorimotor networks for auditory-motor coupling.
\end{abstract}

\section{Introduction}

Neural representation of temporal information is essential for performing rhythmic movements. A privileged connection between auditory and motor systems has been shown in entraining temporal information and using it for motor planning when subjects consciously perform a repetitive movement task in synchrony with external stimuli (for review, see Repp, 2005). Movement timing with a metronome beat becomes less variable and is increasingly based on prediction especially around the rate of 1-3 Hz (Toma et al., 2002), showing clear facilitation effects of the sound. The $1-3 \mathrm{~Hz}$ frequency range coincides with the optimal range of musical tempo in auditory perception (Large, 2008). Neural processing for timing in this range also appears to involve both basal ganglia and cerebellum (Ivry and Spencer, 2004; Mauk and Buonomano, 2004). However, the neural mechanisms un-

Received Aug. 9, 2011; revised Nov. 10, 2011; accepted Dec. 8, 2011.

Author contributions: T.F., L.J.T., E.W.L., and B.R. designed research; T.F. and B.R. performed research; T.F. and B.R. contributed unpublished reagents/analytic tools; T.F. and B.R. analyzed data; T.F., L.J.T., E.W.L., and B.R. wrote the paper.

This work was supported by Canadian Institutes of Health Research Grant 81135 (B.R.), the Centre for Stroke Rehabilitation of the Heart and Stroke Foundation of Ontario (T.F.), and the Canadian Foundation for Innovation. We sincerely thank Drs. William D. Hutchison and Norihiro Sadato for the insightful comments on an earlier version of this manuscript.

The authors declare no competing financial interests.

Correspondence should be addressed to Dr. Takako Fujioka, Rotman Research Institute, 3560 Bathurst Street, Toronto, 0N M6A 2E1, Canada. E-mail: tfujioka@rotman-baycrest.on.ca.

DOI:10.1523/JNEUROSCI.4107-11.2012

Copyright $\odot 2012$ the authors $\quad 0270-6474 / 12 / 321791-12 \$ 15.00 / 0$ derlying auditory-motor facilitation are poorly understood. In functional magnetic resonance imaging (fMRI) studies, auditory-guided or self-paced repetitive motions (Rao et al., 1997; Jäncke et al., 2000; Chen et al., 2008) and listening to and encoding auditory rhythms internally (Grahn and Brett, 2007; Chen et al., 2008) similarly activated primary sensorimotor cortex (SM1), premotor cortex (PMC), supplementary motor area (SMA), striatum, and cerebellum. Thus, auditory processing for timing may rely on a distributed sensorimotor network, which would enable auditory-motor facilitation.

Here, we hypothesized that beta-band activity $(\sim 20 \mathrm{~Hz})$ may play an important role in functional auditory-motor communication even when no movement is performed. Beta oscillations in central and peripheral sensorimotor systems (Murthy and Fetz, 1992; Sanes and Donoghue, 1993; Baker et al., 1997; Courtemanche et al., 2002, 2003) show dynamic modulation representing active states of the system (Engel and Fries, 2010). In human electroencephalography (EEG) and magnetoencephalography (MEG), beta amplitude decreases before and during a movement, followed by a recovery after task completion (Pfurtscheller, 1981; Salmelin et al., 1995). In our previous work investigating auditory processing of isochronous metronome beats without movement tasks, the signal power of the induced beta oscillations in bilateral auditory cortices was periodically modulated at the rate of the metronome (Snyder and Large, 2005; Fujioka et al., 2009). In a similar manner, beta activity in SM1 and SMA was modulated during repetitive finger motion (Gerloff et al., 1998; Toma et al., 2002). Beta phase coherence during auditory-paced motion has 
been found as another index of functional connectivity between primary auditory cortex (A1), SM1, SMA, cerebellum, thalamus, PMC, and posterior parietal cortex (Pollok et al., 2005). However, the contribution of auditory input to this network and the dynamics of linkages across regions have not been investigated.

Thus, the current study investigated the dynamics of beta activity to examine auditory-motor communication. Specifically, we tested the hypotheses that (1) periodic modulation of induced beta activities would anticipate acoustic events, providing a predictive temporal representation of stimulus rate, and that both the beta (2) power comodulation and (3) phase-coherence would span auditory- and motor-related brain areas, providing evidence for long-range communication (Kopell et al., 2000).

\section{Materials and Methods}

Participants. Twelve healthy right-handed young adults (age, 22-33; seven females) participated in the study. None reported any history of neurological or psychological disorders. Normal hearing between 250 and $4000 \mathrm{~Hz}$ was confirmed with clinical audiometry. The Research Ethics Board at Baycrest Centre approved the study, and participants signed consent forms after written information about the study had been provided.

Stimuli. Auditory stimuli were $262 \mathrm{~Hz}$ pure tones with $10 \mathrm{~ms}$ rise and fall times and $40 \mathrm{~ms}$ steady-state duration. In the first three conditions, sounds were rhythmically presented with onset-to-onset intervals of 390 , 585 , and $780 \mathrm{~ms}$, equivalent to stimulus rates of $\sim 2.5,1.7$, and $1.3 \mathrm{~Hz}$. In the fourth condition, the stimuli were presented at random onset-toonset intervals chosen uniformly between 390 and $780 \mathrm{~ms}$. Sequences of tones were presented in short trains of $7 \mathrm{~s}$ duration, containing 18, 12, and 9 tones for the regular conditions, respectively, and $\sim 12$ tones for the irregular condition. Trains were separated by $3 \mathrm{~s}$ of silence. Stimulus trains of the same type were presented in blocks of $\sim 8$ min duration. The total number of stimuli per block was $900,600,450$, and 600 , respectively. All conditions were repeated twice with the order of the blocks randomized across participants. The stimulus intensity was set to 60 $\mathrm{dB}$ above individual sensation threshold, which was measured immediately before each MEG recording. All stimulus sequences were presented under control of STIM software (NeuroScan). The sound was delivered binaurally through Etymotic ER3A transducers connected with $1.5 \mathrm{~m}$ of matched plastic tubing and foam insert earphones to the participant's ears.

MEG recording. Neuromagnetic activity was recorded with a 151 channel whole-head axial gradiometer type MEG system (OMEGA; VSM MedTech) in a quiet magnetically shielded room. The MEG was recorded continuously with a sampling rate of $1250 \mathrm{~Hz}$ after $400 \mathrm{~Hz}$ low-pass filtering. Participants were comfortably seated in an upright position with their head resting in the helmet-shaped MEG device and their arms on the arm rests of the chair to avoid any body movements. They were instructed to pay no particular attention to the sounds while watching a subtitled movie of their choice. This is a common practice to maintain participants in an alert state over the time course of a long EEG/MEG experiment investigating automatic sensory functions with passive stimulus experience. Since the participants did not perform any tasks throughout the recording session, there was no explicit association between the auditory stimuli and actions. The compliance of the participants was verified by video monitoring with the visual field covering the arms, hands, and legs to ensure they did not move any body parts in a visible manner. The position of the head relative to the MEG sensor was measured before and after each recording block. Only blocks with head movement $<5 \mathrm{~mm}$ were accepted for the further data analysis.

Data analysis. We used two types of source analysis. First, to examine the behavior of auditory induced beta oscillations in bilateral auditory cortices, we obtained a dipole source model and examined the time series of auditory evoked responses and its time-frequency decomposition representing event-related changes in oscillatory activity. Second, to examine functional communication between auditory and sensorimotor systems, we applied a model-free source analysis using a spatial filter based on a MEG beamformer to investigate beta activity across the whole brain. For these data, we used two measures of functional communication based on beta oscillations: first, the comodulation of the magnitudes of oscillation across the whole brain; and second, the temporal changes in coherence specifically linked to the auditory and sensorimotor cortices, and supplementary motor areas.

Beta oscillations in auditory cortices based on dipole source analysis. For all analyses, we segmented the MEG data into $1900 \mathrm{~ms}$ stimulus-related epochs beginning $800 \mathrm{~ms}$ before stimulus onsets. Eye blink artifacts were eliminated using principal component analysis (PCA) in which components exceeding the threshold of $1.5 \mathrm{pT}$ at any MEG sensor were subtracted. From time domain averaging of the data in each experimental block, we obtained the auditory evoked responses. Visual inspection of the auditory evoked response revealed that there was no baseline shift before the stimulus onset. This means that there was no sensorimotor evoked response prior to the sound onset, thus indicating the absence of any synchronized movements (see Fig. $1 E$ ). The magnetic field maps showed exclusively dipolar patterns of the auditory evoked response, which were modeled with equivalent current dipoles in left and right auditory cortices. Specifically, the locations and orientations of the dipoles were estimated for the $\mathrm{P} 1 \mathrm{~m}$ peak around $80 \mathrm{~ms}$ after stimulus onset because at the fast stimulus rates in our experiment $(>1 / \mathrm{s})$ the P1m was the most dominant peak whereas the $\mathrm{N} 1 \mathrm{~m}$ response was strongly reduced (Näätänen and Picton, 1987) (see Fig. 1E). Based on individual dipole models, the source activities of single trials in auditory cortices were extracted from all epochs using signal space projection.

Time-frequency decompositions were calculated for each subject on each single-trial source waveform in left and right auditory cortices and for each stimulus condition using a Morlet wavelet transform (Bertrand et al., 1994). The wavelet was designed such that the half-maximum width was equal to 2 periods of the center frequency (e.g., $100 \mathrm{~ms}$ at 20 $\mathrm{Hz}$ ). The wavelet transform was calculated for each time point between -800 and 1100 ms with 128 frequency bins between 6 and $100 \mathrm{~Hz}$. The further analysis eliminated both ends of the data segment by $200 \mathrm{~ms}$ to avoid aliasing caused by the time-frequency analysis. The mean signal power was calculated at each frequency bin as the mean variance of wavelet coefficients across trials. Normalizing this to its mean value across the whole epoch for each frequency resulted in relative signal power changes expressed in percentage and was visualized as function of time and frequency in color-coded maps of event-related synchronization (ERS) and desynchronization (ERD) (Pfurtscheller and Lopes da Silva, 1999). Time courses of beta power change were calculated as the mean across the frequencies from 13 to $25 \mathrm{~Hz}$. To describe the onset and offset of beta modulation, we measured the latency of the maximum beta decrement and the latencies of the midpoints of the falling and rising slopes of beta power change for each condition. Bootstrap resampling was used to estimate the $95 \%$ confidence intervals of the group mean latencies. For this procedure, we treated the datasets as independent samples because the noise was independent from trial to trial.

Beta oscillations across brain based on beamformer source analysis. For calculating temporally correlated beta power modulation across the whole brain, we used a two-step procedure. First, a spatial filter algorithm, a beamformer called synthetic aperture magnetometry (SAM), was applied to the magnetic field data to calculate the time series of local source activity at $8 \times 8 \times 8 \mathrm{~mm}$ volume elements covering the whole brain. The SAM approach uses a linearly constrained minimum variance beamformer algorithm (Van Veen et al., 1997; Robinson and Vrba, 1999), normalizes source power across the whole cortical volume (Robinson, 2004), and is capable of revealing deep brain sources (Vrba and Robinson, 2001; Vrba, 2002). SAM source analysis has been successfully applied for identifying activities in auditory (Ross et al., 2009) and sensorimotor cortices (Jurkiewicz et al., 2006), and deep sources such as 
Table 1. Stereotaxic coordinates of brain areas with a statistically significant stimulus-related modulation of beta intensity

\begin{tabular}{|c|c|c|c|c|c|c|}
\hline Condition & Talairach coordinates: LR, PA, IS (mm) & Hemisphere & Labels & Intensity & $t(10)$ & $p$ value \\
\hline \multirow[t]{16}{*}{$390 \mathrm{~ms}$} & $45,-19,14$ & R & Heschl's gyrus, insula, superior temporal gyrus & 0.045 & 4.73 & 0.0011 \\
\hline & $2,-4,72$ & & Medial frontal gyrus (SMA) & 0.043 & 5.40 & 0.0004 \\
\hline & $-34,-27,64$ & L & Precentral, postcentral gyrus & 0.042 & 4.65 & 0.0012 \\
\hline & $38,-27,62$ & $\mathrm{R}$ & Precentral, postcentral gyrus & 0.039 & 3.57 & 0.0060 \\
\hline & $-41,-24,12$ & L & Heschl's gyrus, insula, superior temporal gyrus & 0.039 & 4.59 & 0.0013 \\
\hline & $31,-70,2$ & $\mathrm{R}$ & Lingual gyrus & 0.034 & 5.28 & 0.0005 \\
\hline & $-48,-8,41$ & L & Precentral gyrus & 0.034 & 8.60 & 0.0000 \\
\hline & $11,-76,-11$ & $\mathrm{~L}$ & Fusiform gyrus & 0.031 & 8.54 & 0.0000 \\
\hline & $-48,18,4$ & L & Inferior frontal gyrus & 0.031 & 5.60 & 0.0003 \\
\hline & $-33,-56,54$ & $\mathrm{~L}$ & Superior parietal lobule & 0.031 & 3.31 & 0.0091 \\
\hline & $-11,52,21$ & $\mathrm{~L}$ & Superior frontal gyrus & 0.030 & 6.81 & 0.0001 \\
\hline & $-26,-75,35$ & L & Precuneus, cuneus & 0.029 & 3.47 & 0.0071 \\
\hline & $37,19,-20$ & $\mathrm{R}$ & Superior temporal gyrus & 0.029 & 4.32 & 0.0019 \\
\hline & $-12,-41,72$ & L & Postcentral gyrus & 0.029 & 3.65 & 0.0054 \\
\hline & $67,-21,-3$ & $\mathrm{R}$ & Superior temporal gyrus & 0.027 & 3.77 & 0.0044 \\
\hline & $24,-53,-33$ & $\mathrm{R}$ & Cerebellum & 0.026 & 3.95 & 0.0033 \\
\hline \multirow[t]{9}{*}{$585 \mathrm{~ms}$} & $45,-12,9$ & $\mathrm{R}$ & Heschl's gyrus, insula, superior temporal gyrus & 0.049 & 9.90 & 0.0000 \\
\hline & $-41,-17,7$ & L & Heschl's gyrus, insula, superior temporal gyrus & 0.047 & 5.51 & 0.0004 \\
\hline & $60,21,26$ & $\mathrm{R}$ & Inferior temporal gyrus & 0.044 & 3.99 & 0.0031 \\
\hline & $53,28,0$ & $\mathrm{R}$ & Inferior frontal gyrus & 0.040 & 8.24 & 0.0000 \\
\hline & $-19,-80,26$ & L & Precuneus, cuneus & 0.037 & 4.57 & 0.0014 \\
\hline & $-19,-27,42$ & L & Cingulate gyrus & 0.032 & 10.12 & 0.0000 \\
\hline & $53,25,30$ & R & Middle frontal gyrus & 0.032 & 5.31 & 0.0005 \\
\hline & $-33,-13,62$ & L & Precentral gyrus & 0.030 & 8.09 & 0.0000 \\
\hline & $-48,13,41$ & L & Middle frontal gyrus & 0.025 & 6.19 & 0.0002 \\
\hline \multirow[t]{5}{*}{$780 \mathrm{~ms}$} & $45,-17,7$ & $\mathrm{R}$ & Heschl's gyrus, insula, superior temporal gyrus & 0.057 & 9.08 & 0.0000 \\
\hline & $-34,-12,9$ & L & Heschl's gyrus, insula, superior temporal gyrus & 0.046 & 6.79 & 0.0001 \\
\hline & $17,-78,19$ & R & Precuneus/cuneus & 0.032 & 6.73 & 0.0001 \\
\hline & $60,11,-21$ & $\mathrm{R}$ & Middle/superior temporal gyrus & 0.031 & 5.89 & 0.0002 \\
\hline & $32,-48,5$ & $\mathrm{R}$ & Parahippocampal gyrus & 0.027 & 4.09 & 0.0027 \\
\hline \multirow[t]{7}{*}{ Random } & $-34,-17,7$ & L & Heschl's gyrus, insula, superior temporal gyrus, lentiform nucleus & 0.060 & 4.81 & 0.0010 \\
\hline & $60,-6,4$ & $\mathrm{R}$ & Insula, Heschl's gyrus, superior temporal gyrus & 0.051 & 3.65 & 0.0053 \\
\hline & $-5,-37,68$ & L & Paracentral lobule/postcentral gyrus & 0.048 & 4.05 & 0.0029 \\
\hline & $3,-66,53$ & $\mathrm{R}$ & Precuneus/cuneus & 0.033 & 3.78 & 0.0044 \\
\hline & $-12,-63,24$ & L & Precuneus/cuneus & 0.033 & 3.45 & 0.0073 \\
\hline & $-5,44,-3$ & L & Anterior cingulate & 0.030 & 4.72 & 0.0011 \\
\hline & $-5,42,50$ & L & Superior frontal gyrus & 0.029 & 5.66 & 0.0003 \\
\hline
\end{tabular}

The locations were identified by spatiotemporal PCA as the first principal component separately in the four conditions. Reported are, for each point, the relative change in intensity, and results of a $t$ test across the group. LR, Left/right; $\mathrm{PA}$, posterior/anterior; IS, inferior/superior.

hippocampus (Riggs et al., 2009) and fusiform gyrus and amygdala (Cornwell et al., 2008). In examining beta oscillation here, the beamformer was constructed specifically based on the covariances within the magnetic field data, for all epochs of $15-25 \mathrm{~Hz}$ bandpass filtered data from -200 to $1000 \mathrm{~ms}$ relative to stimulus onset. Also to construct the SAM spatial filter, we used a template brain magnetic resonance image (MRI) (Holmes et al., 1998), which was transformed to Talairach standard coordinates (positive axes toward anterior, right, and superior direction) using the AFNI software package (Cox, 1996). It has been shown recently with simulations and actual data that MEG source analysis based on individual coregistration with a spherical head model and group analysis based on a template brain is reasonably accurate (Steinstraeter et al., 2009). Specifically, their study showed that the localization errors were not larger than the typical spatial uncertainty in group analysis based on Talairach normalization of an order of $\sim 5 \mathrm{~mm}$ in the visual cortex (Hasnain et al., 1998). Accordingly, MEG source localization based on a template brain MRI has been used as the best approximate when the individual MRI is not available (Jensen et al., 2005; Ross et al., 2009; Fujioka et al., 2010).

Time series of ERD/ERS were calculated from the mean signal power in the beta band at each volume element using the SAM-filtered singletrial data, similar to the described analysis of beta oscillations in the auditory cortex source activity. Second, we applied a spatiotemporal PCA to extract the source locations of temporally correlated beta amplitude modulation across the whole brain volume. The PCA decomposed the four-dimensional source data into principal components (PCs), each of which consisted of a spatial map of sources (factor loading) sharing the same time course (factor score) of modulation of beta power. The normalized intensity at each location in the map indicated how strongly the identified time course is observed at that location. PCA results in PCs that are ordered according to the amount of variance explained in the data. The first PC, which accounted for the most of the signal variance, was used for further analysis as the most representative time course of the beta power modulation across brain volume. The variance accounted for by the first PC was $45 \%$ or more across conditions in each individual case. For demonstrating consistency across the group, we applied the described PCA to individual data and compared the time courses of factor scores. Local maxima in the group mean factor-loading map were identified, and a $t$ test was applied to the corresponding individual factor loads at the identified source location to examine whether it was significantly different from zero across the group. Locations with values of $p<$ 0.001 were accepted as significant.

Event-related changes in beta coherence referenced to auditory and motor sources. To characterize possible functional communication between auditory and motor areas, we were interested in cortico-cortical coherence at beta frequencies across the brain volume specifically from the five seed locations in bilateral A1 and SM1, and SMA, as shown in previous findings of betaband coherence in auditory-guided repetitive movements (Pollok et al., 2005). Instead of taking a list of a priori-defined locations as the seed locations, we used the five areas, which were identified in our analysis of the beta 

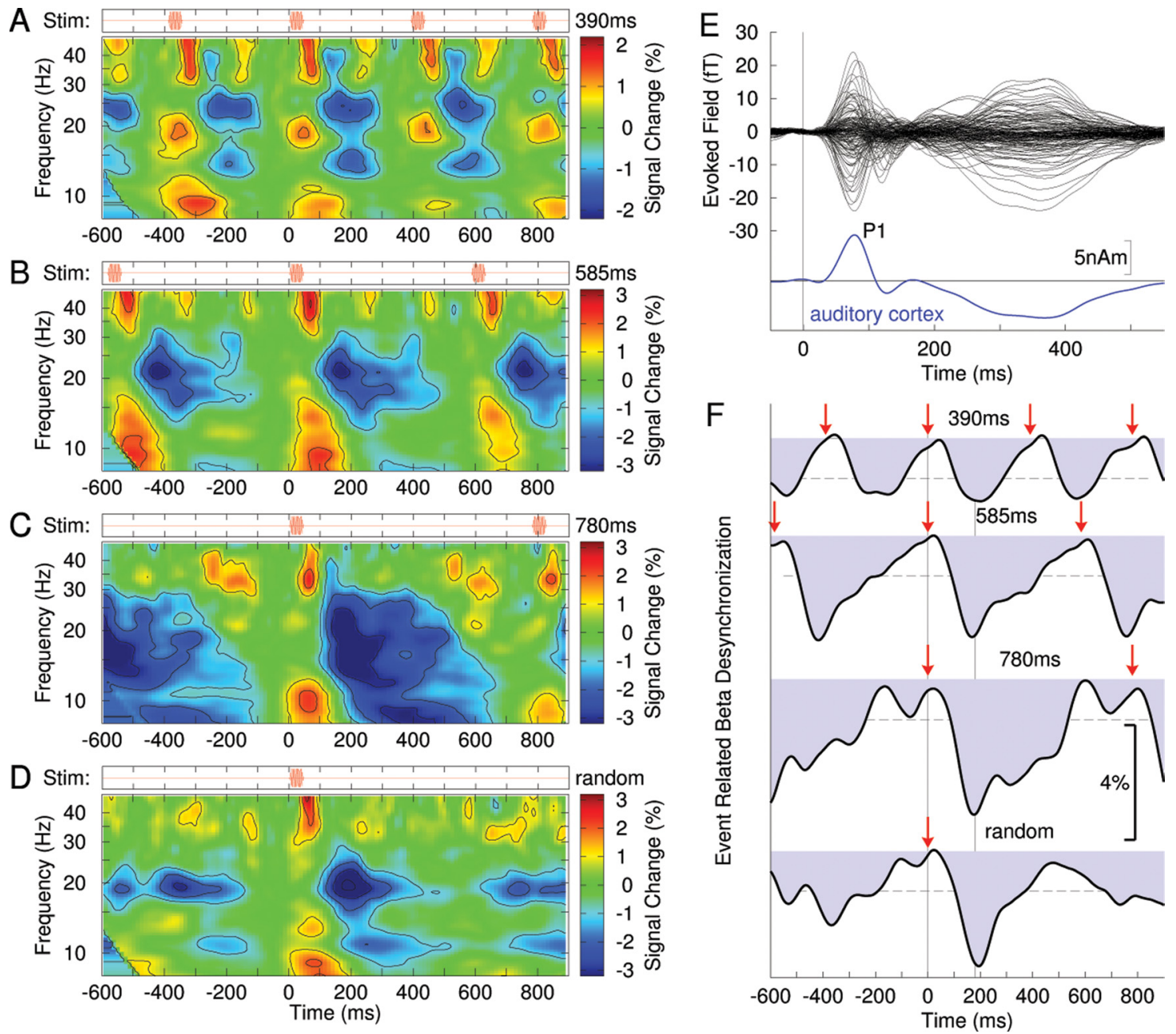

Figure 1. Spectrotemporal dynamics of neuromagnetic activity recorded from right auditory cortex. All data shown are grand averages across the group of 12 participants. $A$, The time-frequency map of signal power changes related to stimuli repeatedly presented every $390 \mathrm{~ms}$ (top row) shows a periodic pattern of signal increases and decreases in various frequency bands. $\boldsymbol{B}$ - $\boldsymbol{D}$, The time-frequency map for stimuli presented every $585 \mathrm{~ms}(\boldsymbol{B})$, every $780 \mathrm{~ms}(\boldsymbol{C})$, and the time-frequency map for randomly presented stimuli (D) show similar stimulus event-related pattern of signal power change. $\boldsymbol{E}$, Auditory evoked magnetic field waveforms observed with $151 \mathrm{MEG}$ channels (top) and the corresponding waveform of auditory cortex activation (bottom). Prominent wave is the $P 1$ response with $\sim 80 \mathrm{~ms}$ peak latency. $F$, Time courses of beta-band power changes $(20-22 \mathrm{~Hz})$ show a signal decrease immediately after stimulus onset, which is termed the beta desynchronization. The red arrows mark the onsets of stimuli. The dashed horizontal lines indicate the $99 \%$ confidence limits for the group mean. The vertical line at $t=190 \mathrm{~ms}$ indicates the latency of largest beta desynchronization, which is similar for all stimulus condition.

amplitude comodulation (390 ms condition) as having the largest PCA factor loading (for coordinates, see Table 1). All source waveforms across the brain volume obtained from the beamformer source analysis in each individual were used to compute beta coherence between a given location and one of the seed locations, defined as follows:

$$
\begin{gathered}
\operatorname{Coh}(x(t), y(t))=\frac{\left\langle G_{x y}(t)\right\rangle^{2}}{\left\langle G_{x x}\right\rangle\left\langle G_{y y}\right\rangle} \\
\left\langle G_{x y}(t)\right\rangle=\frac{1}{N} \sum_{i=1}^{N} x_{i}(t) \cdot y_{i}(t)
\end{gathered}
$$

The complex waveforms $x(t)$ and $y(t)$ were obtained with bandpass filtering between 18 and $25 \mathrm{~Hz}$, and the Hilbert transform was applied to all single trials $(i=1 \ldots N)$ of source waveforms. The coherence is a complex measure of phase synchrony between the waveforms $x(t)$

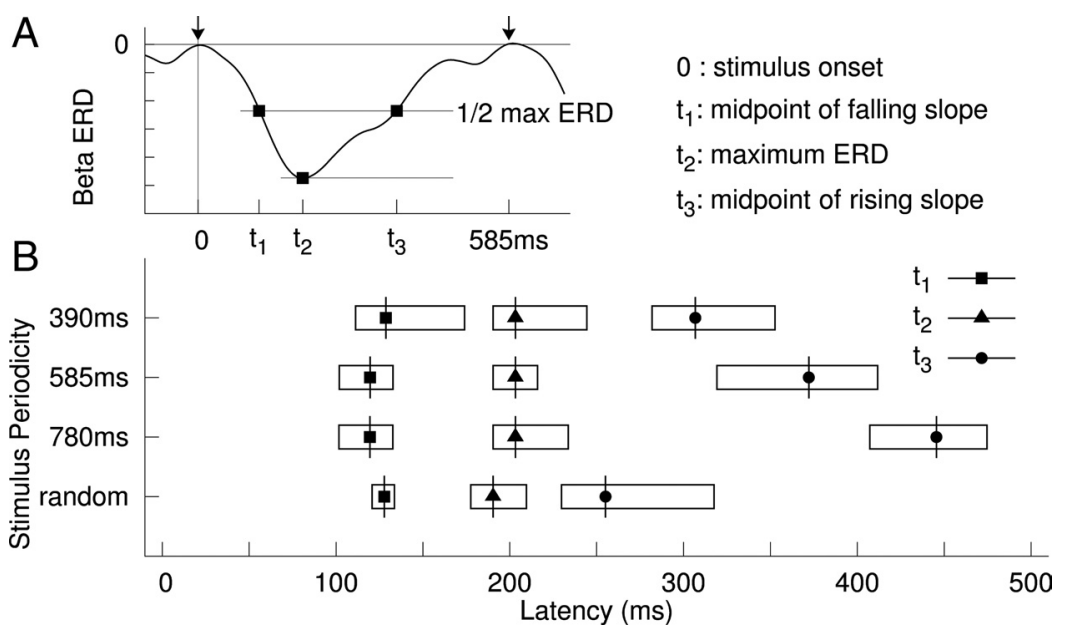

Figure 2. Latency of stimulus-related beta desynchronization. $\boldsymbol{A}$, Schema for measuring the peak latency and the latencies of the midpoints of falling and rising slopes. $\boldsymbol{B}$, Group mean latencies and their $95 \%$ confidence limits indicated by the length of the horizontal bars. 

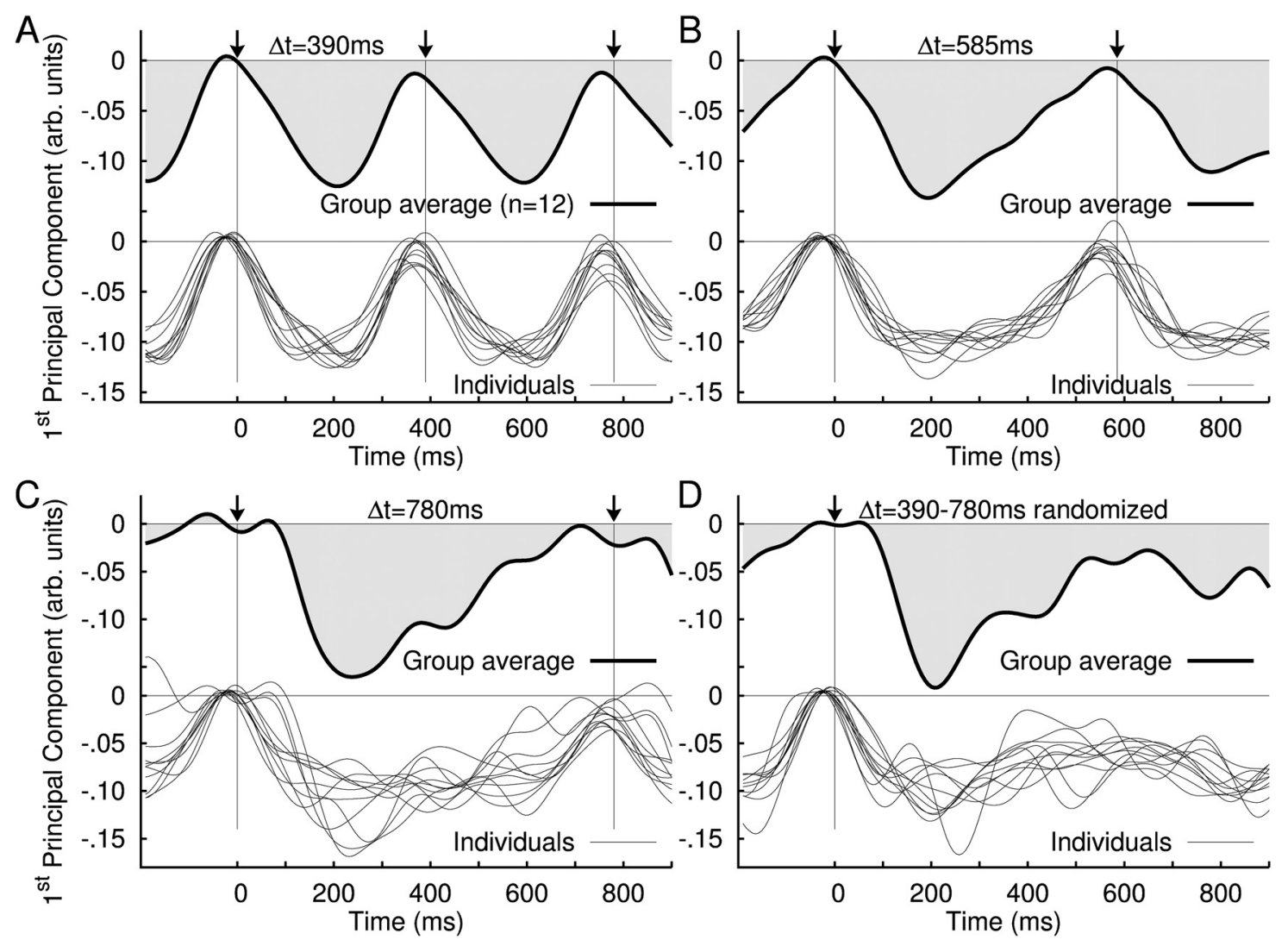

Figure 3. Time series of the principal components of event-related modulation of beta oscillations. A spatiotemporal principal component analysis decomposed the source data of beta signal change into a set of $P C$, consisting of a brain map and associated time series. The time series of the first $P C$ (i.e., the largest) are shown in this figure. $A-D, 0$ verlay of the time series of the first $P C$ for all individuals at 390 ms stimulus onset asynchrony (SOA) (bottom) and the first PC of the grand averaged data (top) (A); 585 ms SOA (B); 780 ms SOA (C); and randomized stimulus presentation $(\boldsymbol{D})$.

and $y(t)$ with its absolute value between 0 and 1 and is calculated for each point in time. However, because the MEG source analysis is limited in spatial resolution, a spread of neural activity across adjacent areas may cause an apparent strong coherence specifically in the vicinity of the seed location. To avoid this, we confined our result to event-related changes in coherence but not the absolute level of the coherence itself. This approach was based on the assumption that functionally relevant coherence fluctuates over time according to the brain process, while apparent coherence due to the spatial filtering should remain constant over the course of time interval of interest rather than dynamically changing with stimulation. Therefore, we subtracted the temporal mean of coherence and analyzed temporal fluctuations only. Moreover, we applied a discrete Fourier transform to the coherence changes and considered only changes that followed the rhythm of the auditory stimulus and termed the resulting measure the event-related coherence. For group statistics, we applied a Rayleigh test (Mardia, 1972; Fisher, 1993), which tests for consistency in the phase of the time course of coherence change across the group.

To reveal the global time courses of event-related coherence, we again applied PCA to decompose the time series of coherence data into principal time series and related volumetric maps. Although the absolute value of coherence measures ranges from 0 to 1 , it decreases with increasing number of trials (Maris et al., 2007), and numerically small coherence values can be highly significant in human EEG and MEG data (Srinivasan et al., 1999; Fries et al., 2001; Tallon-Baudry et al., 2001). According to phase statistics (Fisher, 1993), the null distribution of the phase coherence statistics is the circular normal distribution, and for large number of trials (e.g., $n>50), p$ values can be obtained as $p=\exp (-n R), R=$ $n \cdot \operatorname{coh}^{2}$. Local maxima of the squared event-related coherence, which were significant according to the Rayleigh test, were accepted as nodes of a network of synchronous beta oscillations. Time series of event-related coherence were shown for the identified locations.

\section{Results}

\section{Beta oscillation in the auditory cortex}

The auditory evoked responses were modeled successfully with a pair of equivalent current dipoles in bilateral temporal lobes. The group mean Talairach coordinates of dipole locations were $(-42$, $-22,2 \mathrm{~mm}$ ) (left) and $(40,-24,2 \mathrm{~mm})$ (right) and correspond to the medial parts of bilateral primary auditory cortices. Timefrequency analysis of activity from these locations revealed periodic patterns of signal power increase and decrease according to the periodicity of the stimuli (Fig. $1 A-D$ ). Power modulation, or ERS/ERD, occurred predominantly in the beta frequency band. The time courses of signal changes in the narrow $20-22 \mathrm{~Hz}$ band shown in Figure $1 \mathrm{~F}$ provide a closer look at its temporal dynamics. Beta activity decreased immediately after stimulus onset, reached a minimum around $200 \mathrm{~ms}$ latency. Notably, the time course of this initial beta-ERD did not change with the stimulus conditions. More importantly, only in the periodic stimulus conditions, the following beta-ERS reached the maximum again around the time of next stimulus presentation (Fig. $1 A-C$ ), whereas in the case of the randomized stimulation the beta-ERD/ ERS was aperiodic and transient in its nature (Fig. 1D). Thus, overall, periodic stimulation resulted in a periodic pattern of beta modulation, and that was not case for the irregular stimulus.

We further examined whether the latency of beta-ERD and the following beta-ERS systematically changed across conditions. As shown in Figure 2, the latencies of the midpoints of the falling slope $\left(t_{1}\right)$ and the troughs of ERD $\left(t_{2}\right)$ did not change as stimulus timing changed. By contrast, the midpoints of the rising slopes $\left(t_{3}\right)$ differed 


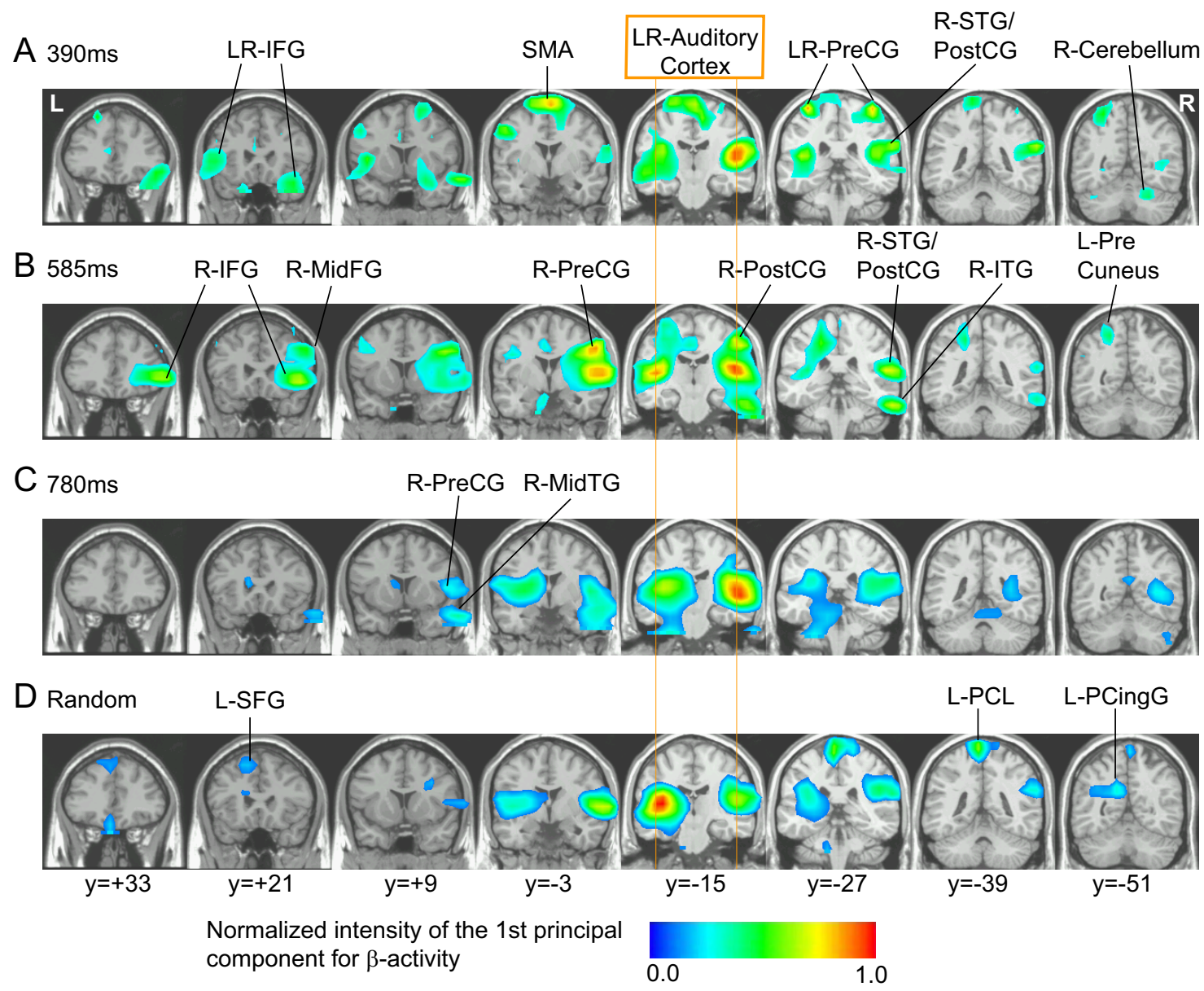

Figure 4. Brain maps associated to the time series of the first $\mathrm{PC}$ of the beta amplitude shown in Figure 3 indicate the areas in which beta activity was modulated by the auditory stimuli. $A-D$, Stimulus interval: $390 \mathrm{~ms}(\boldsymbol{A}), 585 \mathrm{~ms}(\boldsymbol{B}), 780 \mathrm{~ms}(\boldsymbol{C})$, and randomized between 390 and $780 \mathrm{~ms}(\boldsymbol{D})$. Local maxima are indicated by arrows. The list of the locations and Talairach coordinates are indicated in Table 1. IFG, Inferior frontal gyrus; ITG, inferior temporal gyrus; MidTG, middle temporal gyrus; PreCG, precentral gyrus; PostCG, postcentral gyrus; $\mathrm{PCingG,}$ posterior cingulate gyrus; $\mathrm{PCL}$, paracentral lobule; SFG, superior frontal gyrus; SMA, supplementary motor area.

significantly across stimulus timing conditions, such that the rising slope was increasingly shallow with increasing stimulus periodicity. Moreover, despite the fact that, in the irregular condition, the intervals were uniformly randomized between 390 and $780 \mathrm{~ms}$, the beta rebound in that condition was significantly earlier compared with the mean of $t_{3}$ across the three periodic conditions. This indicates that the periodic pattern of beta modulation was only observed with periodic stimulation and it suggests further that, when the time of the next auditory beat is uncertain, the system prepares for the possibility of an early beat rather than simply expecting the next sound to occur at the mean time of previous beats.

The amount of amplitude change was largest for the slowest stimulus rates; group mean ERD maxima and its SD were $1.63 \pm$ $0.25 \%$ at $390 \mathrm{~ms}, 2.68 \pm 0.45 \%$ at $585 \mathrm{~ms}$, and $3.52 \pm 0.52 \%$ at $780 \mathrm{~ms}$. Pairwise comparisons between the different rates revealed significant differences between the fastest condition (390 $\mathrm{ms}$ ) and each of the slower conditions (390 vs $585 \mathrm{~ms}, t_{(17)}=$ $2.033, p=0.029$; 390 vs $780 \mathrm{~ms}, t_{(16)}=3.298, p=0.023$; for this comparison, the sample size of $N=12$ was corrected based on the Welch-Satterthwaite equation to account for unequal number of trials in each condition). There was no significant difference between the two slower conditions, partly reflecting larger residual noise because of the smaller number of trials.

As for the baseline level of beta amplitude, we compared the mean beta amplitude during the $2.0 \mathrm{~s}$ interval preceding a stim- ulus train to that during the stimulus presentation, and found no significant differences between conditions.

\section{Beta power comodulation across the whole brain}

From spatiotemporal PCAs for each stimulus condition, we identified the first PC with its factor score, which reflected common temporal patterns of source activity in the beta power, and its corresponding factor-loading map of source locations, which indicated the strength of the identified temporal pattern at each volume element. Figure 3 illustrates the time series of the first PC, the most common activity across the brain, for the group averaged and individual data. The first PC explained the majority of the variance in beta-power changes for each individual and all conditions (390 ms, 54-80\%; $585 \mathrm{~ms}, 47-75 \%$; $780 \mathrm{~ms}, 47-77 \%$; random, 52-78\%). Furthermore, the time courses of the first PC were consistent across individuals (Fig. 3, bottom panel of each plot), especially for the faster tempi. For the slowest tempo and the randomized tones, the interindividual variability in the time course increased particularly for the upward slope after the initial decrease around 100-300 ms. This apparent larger variability likely reflects the smaller number of trials in these conditions compared the fastest rate condition. However, importantly, this did not affect the timing of the beta rising slope as indicated by similar confidence intervals for the midpoints of the rising slope (Fig. 2). The corresponding map of brain areas in which the beta 

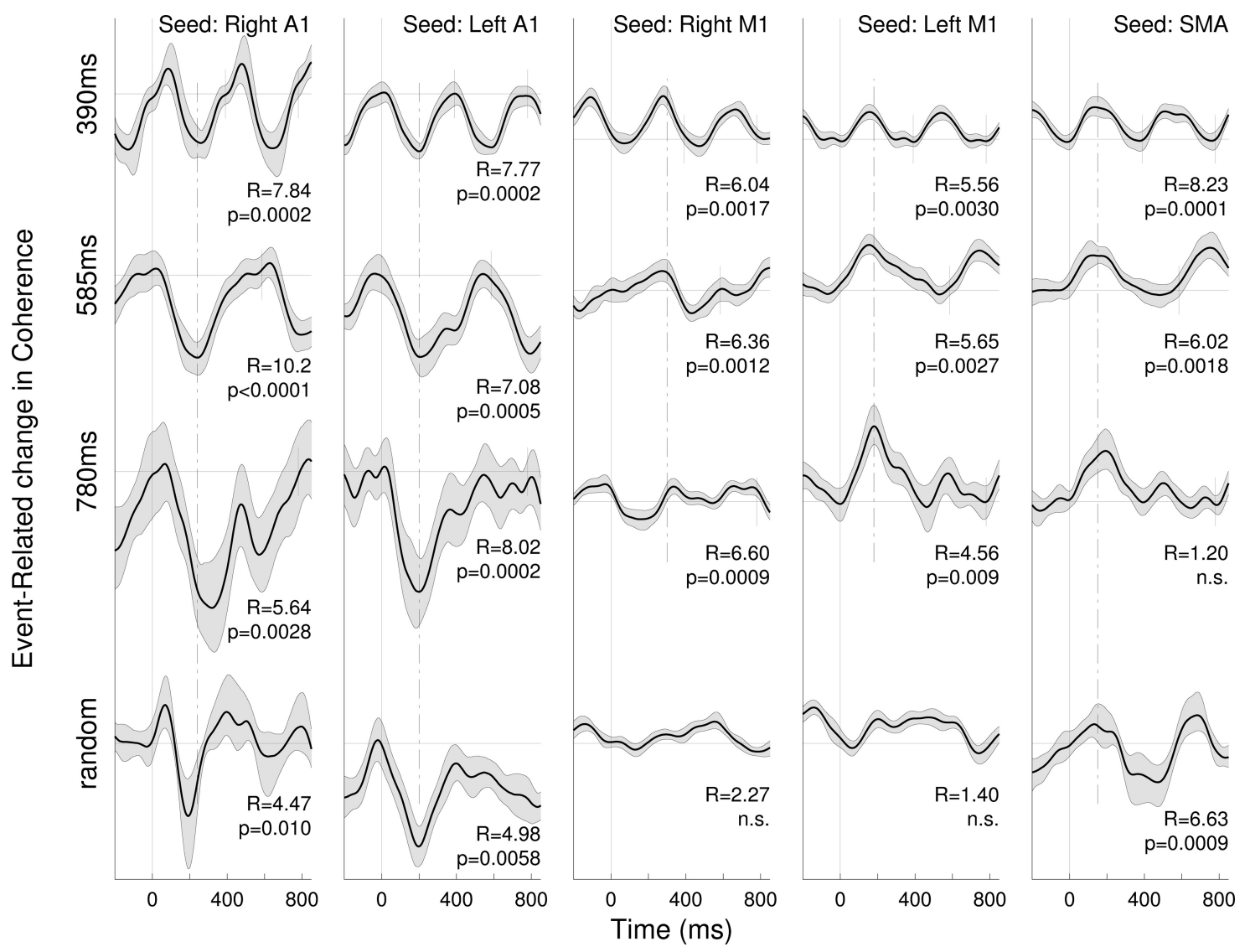

Figure 5. Time series of event-related changes in cortico-cortical coherence of beta oscillations. Shown are the largest principal components resulting from spatiotemporal PCA on the coherence measure. Columns of time series are related to the seed locations for coherence analysis in the right Heschl's gyrus (A1) (Talairach coordinates: 45, $-19,14 \mathrm{~mm}$ ), left Heschl's gyrus ( $-41,-24,12)$, right precentral gyrus $(38,-27,62)$, left precentral gyrus $(-34,-27,64)$, and medial frontal gyrus, location of supplementary motor areas $(2,-4,72)$. Rows of time series relate to the different stimulus periodicity. The gray shaded areas denote the $95 \%$ confidence intervals for the group mean. The Rayleigh statistics $R$ and corresponding $p$ values indicate phase-locking of coherence changes to the stimulus.

decrease was statistically significant $(p<0.001)$ is illustrated in Figure 4 . The complete list of Talairach coordinates of local maxima in the factor-loading map and the $p$ values is given in Table 1. The beta network identified by the first PC involved the bilateral auditory cortices [superior temporal gyrus (STG) and/or Heschl's gyrus (HG)] and cuneus/precuneus for the three stimulus rates consistently. Inclusion of motor-related areas became evident mainly for the two faster stimulation rates. The spatial maps for the two fastest rates included the SM1 (precentral and/or postcentral gyrus) and inferior frontal gyrus (IFG). In the fastest tempo, additional motor regions in SMA and cerebellum were also significant. In contrast, parahippocampal gyrus and middle temporal gyrus participated in the beta modulation in the slowest condition only. In the randomized condition, the posterior cingulate gyrus and superior frontal gyrus (SFG) contributed to the beta network. It is important to note that these locations are limited to the areas mostly contributing to the first PC of the primary beta-power time course described here, while beta oscillations across the whole brain involve other brain areas such as those described below in the beta coherence.

\section{Cortico-cortical beta phase synchrony}

To examine functional communication between the auditory and the motor systems, the time courses of event-related changes in phase coherence across the whole brain were calculated with five seed areas in bilateral A1, bilateral SM1, and SMA for all conditions. In this analysis, we again applied PCA to extract the first PC as the temporal factor score and the corresponding factor-loading map for each of the event-related coherence data with each of the five different seeds in each stimulus condition. Figure 5 indicates the time course of the factor scores showing periodic patterns of beta coherence modulated with the stimulus rhythm in all conditions. Interestingly, coherence with right and left primary auditory cortex areas was characterized by a decrease following stimulus onset. Thus, it appears that the time series of change in coherence resembled closely the modulation of the beta amplitude. In contrast, coherence with sensorimotor areas increased after stimulus onset. It is important to note that eventrelated coherence showed maxima/minima at almost the same latency consistently across all periodic stimulation (Fig. 5, dashed lines).

A snapshot of the spatiotemporal pattern of these coherence data is shown for the seed in the right HG in Figure 6, in which the brain areas were identified as significantly involved in stimuluslocked fluctuation in coherence. Strongest coherence changes were found in auditory areas close to the seed (Fig. 6B) and the left cingulate gyrus, likely related to SMA and/or pre-SMA (Fig. $6 \mathrm{~A}$ ). Further local maxima were found in the right postcentral gyrus, left anterior cingulated gyrus, left inferior temporal gyrus, and right parahippocampal gyrus (Fig. 6C-F).

The complete list of brain areas connected through eventrelated coherence changes is indicated in Table 2. As shown in 

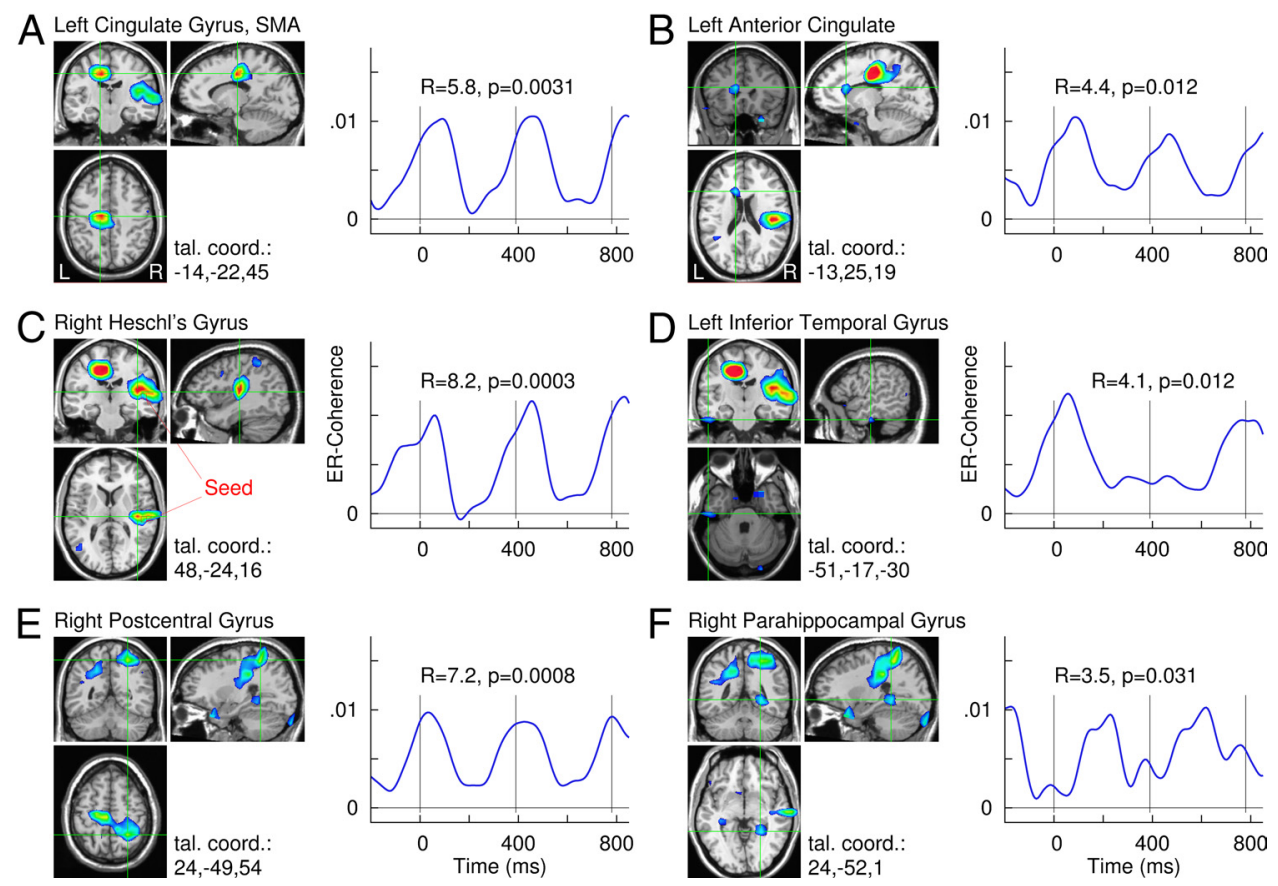

Figure 6. Brain areas identified by event-related changes in beta coherence with the seed in the right Heschl's gyrus. $\boldsymbol{A}-\boldsymbol{F}$, Color-coded maps overlaid to a standard brain MRI show the Rayleigh statistics $R$, which indicates consistent changes in coherence according to the rhythm of auditory stimulation at periodicity of $390 \mathrm{~ms}$. Brain areas with the largest local maxima of $R$ values are shown with axial, coronal, and sagittal views, respectively. Corresponding time series of event-related change in beta coherence with the seed location are shown for the six local maxima. The time series exhibit periodic pattern according to the stimulus rhythm. The vertical lines indicate the onsets of auditory stimuli.

the list, ipsilateral connections to STG, middle/inferior frontal gyrus, and inferior parietal lobules and contralateral connections to medial frontal gyri were found with seed locations in primary auditory cortices. However, no significant coherence between left and right auditory cortices was found. More importantly, if the seed location was in the sensorimotor areas, coherence was mainly significant with motor-related areas, but not in the auditory areas.

\section{Discussion}

The current study revealed three main findings regarding beta oscillations induced through passive listening to isochronous sound stimuli. First, auditory cortices showed a periodic beta modulation consisting of an initial rapid beta decrease, immediately following the stimulus onset and the subsequent rebound. Importantly, only the latter depended on the stimulus rate in the regular conditions, suggesting representation of the internalized interval. Second, the brain areas showing temporally correlated beta modulation with that in the auditory cortices included motor-related cortical and subcortical areas even in the absence of movement. Third, neural synchrony measured as corticocortical phase coherence at beta frequencies, was modulated with the sound rhythm in a consistent manner, also spanning across auditory and motor systems.

\section{Rebound of beta activity as representation of internalized timing}

The rising slope of the beta rebound but not the initial falling slope varied according to the stimulus rate in the auditory cortex as well as across an extensive brain network. This suggests an endogenous nature of the beta rebound, in line with our previous MEG study (Fujioka et al., 2009) in which a beta decrement in the auditory cortex was absent after an occasional stimulus omission whereas the subsequent rising slope was not affected until the timing of the omission. After the omission, the rising slope continued toward the next stimulus onset. Thus, in both studies, we observed that the amplitude of beta oscillation peaked just before the next stimulus during isochronous stimulation. The faster the tempo of the stimulus, the steeper the rising slope became. Thus, the beta rebound may reflect a neural mechanism for predictive timing information about when the next stimulus is expected. This is a sharp contrast to the case in the irregular condition in which the slope was significantly shorter than in any of the regular conditions and much shorter than expected from the mean of the onset-to-onset intervals. Thus, the predictable periodic characteristic of the auditory input is critical to the beta dynamics described here. Nevertheless, the beta rebound after the initial decrease in the irregular condition may also indicate some anticipatory process because the next stimulus can be still expected within the narrow time window.

Recent studies also associated induced beta increase with endogenous processes: internally driven choices in a search task (Buschman and Miller, 2009), anticipation to the predictive timing of the task-relevant cue (Saleh et al., 2010), and top-down attentional processing in ambiguous visual perception (Okazaki et al., 2008). Our results particularly resemble those of human intracranial recordings in which the beta increase peaked just before an informative cue presented with predictable timing (Saleh et al., 2010). Thus, extending our previous findings (Snyder and Large, 2005; Fujioka et al., 2009), here we propose that the observed time course of beta rebound likely reflects endogenous processing of internalized timing.

\section{Dissociation between beta suppression and rebound}

In contrast to the beta rebound, the initial beta decrease after the stimulus onset was almost identical regardless of the regularity or rate of the stimulus sequence. This suggests that this beta decrease reflects an exogenous process driven by the stimulus input, as 
Table 2. Stereotaxic coordinate of locations with largest event-related modulation of cortico-cortical coherence

\begin{tabular}{|c|c|c|c|c|c|}
\hline Seed & Talairach coordinates: LR, PA, IS (mm) & Hemisphere & Labels & $R$ & $p$ value \\
\hline \multirow[t]{8}{*}{ Right A1 } & $63,-21,2$ & $R$ & Superior temporal gyrus & 7.06 & 0.0009 \\
\hline & $40,-24,15$ & $\mathrm{R}$ & Heschl's gyrus & 7.05 & 0.0009 \\
\hline & $-14,-14,47$ & $\mathrm{~L}$ & Medial frontal gyrus, left SMA & 7.09 & 0.0009 \\
\hline & $55,-44,-4$ & $\mathrm{R}$ & Middle/inferior frontal gyrus & 6.08 & 0.0023 \\
\hline & $39,-62,36$ & $\mathrm{R}$ & Inferior parietal lobule & 8.29 & 0.0003 \\
\hline & $33,4,67$ & $\mathrm{R}$ & Middle/superior frontal gyrus & 6.78 & 0.0011 \\
\hline & $-21,1,-10$ & L & Parahippocampal gyrus & 3.50 & 0.030 \\
\hline & $17,-42,-2$ & $\mathrm{R}$ & Parahippocampal gyrus & 5.16 & 0.0057 \\
\hline \multirow[t]{8}{*}{ Left A1 } & $-37,-27,36$ & $\mathrm{~L}$ & Inferior parietal lobe & 5.46 & 0.0042 \\
\hline & $-52,-19,37$ & $\mathrm{~L}$ & Precentral/postcentral gyrus & 6.30 & 0.0018 \\
\hline & $-29,-67,3$ & $\mathrm{~L}$ & Middle occipital gyrus & 5.55 & 0.0039 \\
\hline & $25,-4,5$ & $\mathrm{R}$ & Putamen, lentiform nucleus & 6.05 & 0.0024 \\
\hline & $-67,-26,5$ & $\mathrm{~L}$ & Superior temporal gyrus & 5.48 & 0.0042 \\
\hline & $2,10,46$ & $\mathrm{R}$ & Medial frontal gyrus, SMA & 4.29 & 0.013 \\
\hline & $-44,-42,1$ & $\mathrm{~L}$ & Middle/inferior temporal gyrus & 3.63 & 0.026 \\
\hline & $-13,4,6$ & L & Putamen, lentiform nucleus & 5.34 & 0.0048 \\
\hline \multirow[t]{8}{*}{ Right M1 } & $18,9,16$ & $\mathrm{R}$ & Caudate, putamen, lentiform nucleus & 6.21 & 0.0020 \\
\hline & $17,-42,56$ & $\mathrm{R}$ & Paracentral lobule, postcentral gyrus & 5.70 & 0.0034 \\
\hline & $-45,-50,0$ & $\mathrm{~L}$ & Middle/inferior temporal gyrus & 5.13 & 0.0059 \\
\hline & $-52,-33,-34$ & $\mathrm{~L}$ & Cerebellum & 3.44 & 0.032 \\
\hline & $32,-21,39$ & $\mathrm{R}$ & Precentral/postcentral gyrus & 6.98 & 0.0009 \\
\hline & $-51,40,-1$ & $\mathrm{~L}$ & Inferior frontal gyrus & 7.69 & 0.0005 \\
\hline & $-22,-48,53$ & L & Precuneus, paracentral lobule & 3.55 & 0.029 \\
\hline & $-21,1,51$ & $\mathrm{~L}$ & Medial frontal gyrus & 5.01 & 0.0066 \\
\hline \multirow[t]{8}{*}{ Left M1 } & $16,-49,54$ & $\mathrm{R}$ & Precuneus, superior parietal lobule & 10.74 & $<0.0001$ \\
\hline & $-13,-7,18$ & $\mathrm{~L}$ & Caudate, left thalamus & 7.38 & 0.0006 \\
\hline & $56,25,13$ & $\mathrm{R}$ & Inferior/middle frontal gyrus & 7.55 & 0.0005 \\
\hline & $-28,-1,-3$ & $\mathrm{~L}$ & Putamen, lentiform nucleus & 5.51 & 0.0040 \\
\hline & $-60,-55,-10$ & L & Inferior/middle temporal gyrus & 3.92 & 0.020 \\
\hline & $33,20,34$ & $\mathrm{R}$ & Precentral gyrus & 6.37 & 0.0017 \\
\hline & $-29,-31,-11$ & $\mathrm{~L}$ & Parahippocampal gyrus, hippocampus & 4.61 & 0.0098 \\
\hline & $-67,-5,-13$ & L & Inferior/middle temporal gyrus & 5.47 & 0.0042 \\
\hline \multirow[t]{8}{*}{ SMA } & $-21,20,40$ & L & Middle/superior frontal gyrus & 5.37 & 0.0046 \\
\hline & $-5,44,39$ & $\mathrm{~L}$ & Medial frontal gyrus & 8.91 & 0.0001 \\
\hline & $-52,4,43$ & L & Precentral gyrus & 5.21 & 0.0054 \\
\hline & $55,-46,-27$ & $\mathrm{R}$ & Culmen, fusiform gyrus & 7.52 & 0.0005 \\
\hline & $63,-25,16$ & $\mathrm{R}$ & Superior temporal gyrus, inferior parietal lobule & 8.97 & 0.0001 \\
\hline & $48,-21,-29$ & R & Inferior temporal gyrus & 4.79 & 0.0082 \\
\hline & $2,-28,6$ & $\mathrm{R}$ & Thalamus & 4.06 & 0.017 \\
\hline & $-29,-26,-32$ & $\mathrm{~L}$ & Culmen, cerebellum & 3.81 & 0.022 \\
\hline
\end{tabular}

Reported are the largest local maxima in brain maps of the Rayleigh statistics $(R)$, indicating consistent coherence changes according to the stimulus rhythms across the group of 12 participants. The statistical maps were calculated for each of the five seed locations as mean across stimulus conditions. LR, Left/right; PA, posterior/anterior; IS, inferior/superior.

opposed to the endogenous nature of the following beta increase described above. The functional difference between beta suppression and rebound found here is in line with the literature. It is widely accepted that beta desynchronization indicates an "active change" in the status of sensorimotor processing (Pfurtscheller and Lopes da Silva, 1999), whereas the beta increase is associated with "maintaining an existing steady state in motor control" (Gilbertson et al., 2005; Pogosyan et al., 2009), "immobilization" (Salmelin et al., 1995), or "reset of an existing cortical network" (Pfurtscheller et al., 2005), reflecting a variety of task and attentional demands. For sensorimotor tasks, slightly different cortical areas seem to be involved in beta suppression and rebound (Salmelin and Hari, 1994; Salmelin et al., 1995; Jurkiewicz et al., 2006; Caetano et al., 2007; Bardouille et al., 2010). Our previous and current findings extend these studies by demonstrating that the difference between suppression and rebound is also manifested in their temporal dynamics.

Using a passive listening paradigm, our study discovered that periodic beta power modulation consisting of these two different processes does not require an explicit motor task. This suggests the possible leading role of the auditory system for propagation of beta oscillation in auditory-guided movement, of which the beneficial effect is found in Parkinson's disease (PD) patients (Freeman et al., 1993; McIntosh et al., 1997). A regular pattern of beta modulation caused by the regular sound might help temporarily alleviate an excessive beta power associated with movement initiation problems in PD (Brown, 2003; Hutchison et al., 2004) by possibly modulating membrane hyperpolarization in thalamic nuclei (Llinás et al., 2005). However, as only a few studies with auditory stimuli have reported beta suppression (Kaiser et al., 2002; Pesonen et al., 2006; Kim and Chung, 2008) and beta increase (Caetano et al., 2007), future research is necessary to examine the nature of beta activities in auditory tasks more in detail. Also, it remains unclear how the observed beta activity is differently influenced by the stimulus interval and its perceptual correlates as the latter is influenced by auditory sequence contexts or individual preference with musical expertise (Jones and McAuley, 2005; Repp and Doggett, 2007). Another remaining question is how the two processes might use different pathways to carry out auditory-sensorimotor communication. A different 
form of auditory-motor transformation such as voice perceptions involving posterior auditory cortices (Warren et al., 2005) could also activate beta modulation, in line with available evidence for speech perception based on articulatory characteristics (Eulitz and Obleser, 2007) and audiovisual multimodal integration (Fingelkurts et al., 2007).

\section{Rate effects on beta modulation in the motor system}

We found temporally correlated beta amplitude modulations in motor-related brain areas and in auditory cortices. There was extended involvement of IFG, SMA, and cerebellum in the beta modulation at faster stimulus rates. To our knowledge, this is the first demonstration of rate-specific dynamic configuration of oscillatory beta networks in passive listening to auditory rhythms. The results are generally in line with previous $\mathrm{PMRI} / \mathrm{PET}$ studies showing that movement rate affects the spatial extent and magnitude of contribution to the activity in different motor regions (Blinkenberg et al., 1996; Rao et al., 1996; Sadato et al., 1996; Schlaug et al., 1996; Jäncke et al., 1998; Wildgruber et al., 2001; Riecker et al., 2003), with decreased activity in the basal ganglia and increased activity in cerebellum and primary motor cortex with increased rate of movements.

\section{Coherence measured by beta phase synchrony}

Our measure of cortico-cortical coherence was limited to eventrelated changes over time, to strictly avoid spatial leakage effects caused by MEG source analysis. This entirely data-driven analysis successfully identified a shared time course of beta coherence that followed the sound rhythm in auditory and sensorimotor networks. Our results indicate that the sensorimotor cortices clearly contributes the modulation of beta coherence with numerous brain areas during passive listening, and that common modulation of synchrony was mainly expressed "within" a modalityspecific neural network (e.g., both for auditory and sensorimotor systems) but less expressed between the two systems and almost absent between left and right auditory areas. Thus, phase synchrony in beta oscillations may play an important role for connectivity within specific functional networks, whereas coupling between networks may rely on different mechanisms. Nevertheless, the most important finding here is that the synchrony was modulated directly by the auditory input across the different brain areas as a function of the stimulus rate. This finding is in agreement with the current model of a functional network that modulation of synchrony is a mechanism of dynamic network reconfiguration (Womelsdorf et al., 2007). Mechanisms underlying the beta dynamics could be a frequency transition of oscillatory circuits through synaptic coupling alterations (Kopell et al., 2000; Jensen et al., 2005), changes in nonsynaptic coupling through gap junction conductance in cortical neurons (Roopun et al., 2006), and synaptic plasticity in cortico-striatum connections (Fino et al., 2005). The latter is particularly interesting in that the striato-thalamo-cortical circuit is involved in coincidence detection of oscillatory activities for temporal coding (Matell and Meck, 2004).

\section{Conclusions}

In conclusion, our findings support the concept of auditorymotor facilitation in that the periodic modulation of beta activity following fast-paced regular auditory stimuli could aid the initiation of movement. More importantly, the beta rebound before the onset of the next beat appears to encode an internal representation of the predictable time interval, which would likely help predictive movement planning in a time-sensitive manner. Phase synchrony of beta oscillations plays a role in configuring motorrelated networks, whereas functional coupling between auditory and motor systems is mainly expressed as correlated changes in beta power. Future studies are necessary to verify the exact nature of sensorimotor interaction expressed in beta processes and to define the precise pathways involved in auditory-motor communication in temporal processing.

\section{References}

Baker SN, Olivier E, Lemon RN (1997) Coherent oscillations in monkey motor cortex and hand muscle EMG show task-dependent modulation. J Physiol 501:225-241.

Bardouille T, Picton TW, Ross B (2010) Attention modulates beta oscillations during prolonged tactile stimulation. Eur J Neurosci 31:761-769.

Bertrand O, Bohorquez J, Pernier J (1994) Time-frequency digital filtering based on an invertible wavelet transform: an application to evoked potentials. IEEE Trans Biomed Eng 41:77-88.

Blinkenberg M, Bonde C, Holm S, Svarer C, Andersen J, Paulson OB, Law I (1996) Rate dependence of regional cerebral activation during performance of a repetitive motor task: a PET study. J Cereb Blood Flow Metab 16:794-803.

Brown P (2003) Oscillatory nature of human basal ganglia activity: relationship to the pathophysiology of Parkinson's disease. Mov Disord 18:357-363.

Buschman TJ, Miller EK (2009) Serial, covert shifts of attention during visual search are reflected by the frontal eye fields and correlated with population oscillations. Neuron 63:386-396.

Caetano G, Jousmäki V, Hari R (2007) Actor's and observer's primary motor cortices stabilize similarly after seen or heard motor actions. Proc Natl Acad Sci U S A 104:9058-9062.

Chen JL, Penhune VB, Zatorre RJ (2008) Listening to musical rhythms recruits motor regions of the brain. Cereb Cortex 18:2844-2854.

Cornwell BR, Johnson LL, Holroyd T, Carver FW, Grillon C (2008) Human hippocampal and parahippocampal theta during goal-directed spatial navigation predicts performance on a virtual Morris water maze. J Neurosci 28:5983-5990.

Courtemanche R, Pellerin JP, Lamarre Y (2002) Local field potential oscillations in primate cerebellar cortex: modulation during active and passive expectancy. J Neurophysiol 88:771-782.

Courtemanche R, Fujii N, Graybiel AM (2003) Synchronous, focally modulated beta-band oscillations characterize local field potential activity in the striatum of awake behaving monkeys. J Neurosci 23:11741-11752.

Cox RW (1996) AFNI: software for analysis and visualization of functional magnetic resonance neuroimages. Comput Biomed Res 29:162-173.

Engel AK, Fries P (2010) Beta-band oscillations-signalling the status quo? Curr Opin Neurobiol 20:156-165.

Eulitz C, Obleser J (2007) Perception of acoustically complex phonological features in vowels is reflected in the induced brain-magnetic activity. Behav Brain Funct 3:26.

Fingelkurts AA, Fingelkurts AA, Krause CM (2007) Composition of brain oscillations and their functions in the maintenance of auditory, visual and audio-visual speech percepts: an exploratory study. Cogn Process 8:183-199.

Fino E, Glowinski J, Venance L (2005) Bidirectional activity-dependent plasticity at corticostriatal synapses. J Neurosci 25:11279-11287.

Fisher NI (1993) Statistical analysis of circular data. Cambridge, UK: Cambridge UP.

Freeman JS, Cody FW, Schady W (1993) The influence of external timing cues upon the rhythm of voluntary movements in Parkinson's disease. J Neurol Neurosurg Psychiatry 56:1078-1084.

Fries P, Neuenschwander S, Engel AK, Goebel R, Singer W (2001) Rapid feature selective neuronal synchronization through correlated latency shifting. Nat Neurosci 4:194-200.

Fujioka T, Trainor LJ, Large EW, Ross B (2009) Beta and gamma rhythms in human auditory cortex during musical beat processing. Ann N Y Acad Sci 1169:89-92.

Fujioka T, Zendel BR, Ross B (2010) Endogenous neuromagnetic activity for mental hierarchy of timing. J Neurosci 30:3458-3466. 
Gerloff C, Richard J, Hadley J, Schulman AE, Honda M, Hallett M (1998) Functional coupling and regional activation of human cortical motor areas during simple, internally paced and externally paced finger movements. Brain 121:1513-1531.

Gilbertson T, Lalo E, Doyle L, Di Lazzaro V, Cioni B, Brown P (2005) Existing motor state is favored at the expense of new movement during $13-35 \mathrm{~Hz}$ oscillatory synchrony in the human corticospinal system. J Neurosci 25:7771-7779.

Grahn JA, Brett M (2007) Rhythm and beat perception in motor areas of the brain. J Cogn Neurosci 19:893-906.

Hasnain MK, Fox PT, Woldorff MG (1998) Intersubject variability of functional areas in the human visual cortex. Hum Brain Mapp 6:301-315.

Holmes CJ, Hoge R, Collins L, Woods R, Toga AW, Evans AC (1998) Enhancement of MR images using registration for signal averaging. J Comput Assist Tomogr 22:324-333.

Hutchison WD, Dostrovsky JO, Walters JR, Courtemanche R, Boraud T, Goldberg J, Brown P (2004) Neuronal oscillations in the basal ganglia and movement disorders: evidence from whole animal and human recordings. J Neurosci 24:9240-9243.

Ivry RB, Spencer RM (2004) The neural representation of time. Curr Opin Neurobiol 14:225-232.

Jäncke L, Specht K, Mirzazade S, Loose R, Himmelbach M, Lutz K, Shah NJ (1998) A parametric analysis of the "rate effect" in the sensorimotor cortex: a functional magnetic resonance imaging analysis in human subjects. Neurosci Lett 252:37-40.

Jäncke L, Loose R, Lutz K, Specht K, Shah NJ (2000) Cortical activations during paced finger-tapping applying visual and auditory pacing stimuli. Brain Res Cogn Brain Res 10:51-66.

Jensen O, Goel P, Kopell N, Pohja M, Hari R, Ermentrout B (2005) On the human sensorimotor-cortex beta rhythm: sources and modeling. Neuroimage 26:347-355.

Jones MR, McAuley JD (2005) Time judgments in global temporal contexts. Percept Psychophys 67:398-417.

Jurkiewicz MT, Gaetz WC, Bostan AC, Cheyne D (2006) Post-movement beta rebound is generated in motor cortex: evidence from neuromagnetic recordings. Neuroimage 32:1281-1289.

Kaiser J, Birbaumer N, Lutzenberger W (2002) Magnetic oscillatory responses to lateralization changes of natural and artificial sounds in humans. Eur J Neurosci 15:345-354.

Kim JS, Chung CK (2008) Language lateralization using MEG beta frequency desynchronization during auditory oddball stimulation with onesyllable words. Neuroimage 42:1499-1507.

Kopell N, Ermentrout GB, Whittington MA, Traub RD (2000) Gamma rhythms and beta rhythms have different synchronization properties. Proc Natl Acad Sci U S A 97:1867-1872.

Large EW (2008) Resonating to musical rhythm: theory and experiment. In: The psychology of time (Grondin S, ed), pp 189-231. Bingley, UK: Emerald.

Llinás R, Urbano FJ, Leznik E, Ramírez RR, van Marle HJ (2005) Rhythmic and dysrhythmic thalamocortical dynamics: GABA systems and the edge effect. Trends Neurosci 28:325-333.

Mardia KV (1972) Statistics on directional data. London: Academic.

Maris E, Schoffelen JM, Fries P (2007) Nonparametric statistical testing of coherence differences. J Neurosci Methods 163:161-175.

Matell MS, Meck WH (2004) Cortico-striatal circuits and interval timing: coincidence detection of oscillatory processes. Brain Res Cogn Brain Res 21:139-170

Mauk MD, Buonomano DV (2004) The neural basis of temporal processing. Annu Rev Neurosci 27:307-340.

McIntosh GC, Brown SH, Rice RR, Thaut MH (1997) Rhythmic auditorymotor facilitation of gait patterns in patients with Parkinson's disease. J Neurol Neurosurg Psychiatry 62:22-26.

Murthy VN, Fetz EE (1992) Coherent 25- to 35-Hz oscillations in the sensorimotor cortex of awake behaving monkeys. Proc Natl Acad Sci U S A 89:5670-5674.

Näätänen R, Picton T (1987) The N1 wave of the human electric and magnetic response to sound: a review and an analysis of the component structure. Psychophysiology 24:375-425.

Okazaki Y, Abrahamyan A, Stevens CJ, Ioannides AA (2008) The timing of face selectivity and attentional modulation in visual processing. Neuroscience 152:1130-1144.
Pesonen M, Björnberg CH, Hämäläinen H, Krause CM (2006) Brain oscillatory 1-30 Hz EEG ERD/ERS responses during the different stages of an auditory memory search task. Neurosci Lett 399:45-50.

Pfurtscheller G (1981) Central beta rhythm during sensorimotor activities in man. Electroencephalogr Clin Neurophysiol 51:253-264.

Pfurtscheller G, Lopes da Silva FH (1999) Event-related EEG/MEG synchronization and desynchronization: basic principles. Clin Neurophysiol 110:1842-1857.

Pfurtscheller G, Muller-Putz GR, Pfurtscheller J, Rupp R (2005) EEG-based asynchronous BCI controls functional electrical stimulation in a tetraplegic patient. EURASIP J Appl Signal Processing 2005:3152-3155.

Pogosyan A, Gaynor LD, Eusebio A, Brown P (2009) Boosting cortical activity at beta-band frequencies slows movement in humans. Curr Biol 19:1637-1641.

Pollok B, Südmeyer M, Gross J, Schnitzler A (2005) The oscillatory network of simple repetitive bimanual movements. Brain Res Cogn Brain Res 25:300-311.

Rao SM, Bandettini PA, Binder JR, Bobholz JA, Hammeke TA, Stein EA, Hyde JS (1996) Relationship between finger movement rate and functional magnetic resonance signal change in human primary motor cortex. J Cereb Blood Flow Metab 16:1250-1254.

Rao SM, Harrington DL, Haaland KY, Bobholz JA, Cox RW, Binder JR (1997) Distributed neural systems underlying the timing of movements. J Neurosci 17:5528-5535.

Repp BH (2005) Sensorimotor synchronization: a review of the tapping literature. Psychon Bull Rev 12:969-992.

Repp BH, Doggett R (2007) Tapping to a very slow beat: a comparison of musicians and nonmusicians. Music Percept 24:367-376.

Riecker A, Wildgruber D, Mathiak K, Grodd W, Ackermann H (2003) Parametric analysis of rate-dependent hemodynamic response functions of cortical and subcortical brain structures during auditorily cued finger tapping: a fMRI study. Neuroimage 18:731-739.

Riggs L, Moses SN, Bardouille T, Herdman AT, Ross B, Ryan JD (2009) A complementary analytic approach to examining medial temporal lobe sources using magnetoencephalography. Neuroimage 45:627-642.

Robinson SE (2004) Localization of event-related activity by SAM(erf). Neurol Clin Neurophysiol 2004:109.

Robinson SE, Vrba J (1999) Functional neuroimaging by synthetic aperture magnetometry. In: Recent advances in biomagnetism (Yoshimoto T, Kotani M, Kuriki S, Karibe H, Nakasato N, eds), pp 302-305. Sendai, Japan: Tohoku UP.

Roopun AK, Middleton SJ, Cunningham MO, LeBeau FE, Bibbig A, Whittington MA, Traub RD (2006) A beta2-frequency (20-30 Hz) oscillation in nonsynaptic networks of somatosensory cortex. Proc Natl Acad Sci U S A 103:15646-15650.

Ross B, Snyder JS, Aalto M, McDonald KL, Dyson BJ, Schneider B, Alain C (2009) Neural encoding of sound duration persists in older adults. Neuroimage 47:678-687.

Sadato N, Ibañez V, Deiber MP, Campbell G, Leonardo M, Hallett M (1996) Frequency-dependent changes of regional cerebral blood flow during finger movements. J Cereb Blood Flow Metab 16:23-33.

Saleh M, Reimer J, Penn R, Ojakangas CL, Hatsopoulos NG (2010) Fast and slow oscillations in human primary motor cortex predict oncoming behaviorally relevant cues. Neuron 65:461-471.

Salmelin R, Hari R (1994) Characterization of spontaneous MEG rhythms in healthy adults. Electroencephalogr Clin Neurophysiol 91:237-248.

Salmelin R, Hämäläinen M, Kajola M, Hari R (1995) Functional segregation of movement-related rhythmic activity in the human brain. Neuroimage 2:237-243.

Sanes JN, Donoghue JP (1993) Oscillations in local field potentials of the primate motor cortex during voluntary movement. Proc Natl Acad Sci U S A 90:4470-4474.

Schlaug G, Sanes JN, Thangaraj V, Darby DG, Jäncke L, Edelman RR, Warach S (1996) Cerebral activation covaries with movement rate. Neuroreport 7:879-883.

Snyder JS, Large EW (2005) Gamma-band activity reflects the metric structure of rhythmic tone sequences. Brain Res Cogn Brain Res 24:117-126.

Srinivasan R, Russell DP, Edelman GM, Tononi G (1999) Increased synchronization of neuromagnetic responses during conscious perception. J Neurosci 19:5435-5448. 
Steinstraeter O, Teismann IK, Wollbrink A, Suntrup S, Stoeckigt K, Dziewas R, Pantev C (2009) Local sphere-based co-registration for SAM group analysis in subjects without individual MRI. Exp Brain Res 193:387-396.

Tallon-Baudry C, Bertrand O, Fischer C (2001) Oscillatory synchrony between human extrastriate areas during visual short-term memory maintenance. J Neurosci 21:RC177(1-5).

Toma K, Mima T, Matsuoka T, Gerloff C, Ohnishi T, Koshy B, Andres F, Hallett M (2002) Movement rate effect on activation and functional coupling of motor cortical areas. J Neurophysiol 88:3377-3385.

Van Veen BD, van Drongelen W, Yuchtman M, Suzuki A (1997) Localization of brain electrical activity via linearly constrained minimum variance spatial filtering. IEEE Trans Biomed Eng 44:867-880.
Vrba J (2002) Magnetoencephalography: the art of finding a needle in a haystack. Physica C 368:1-9.

Vrba J, Robinson SE (2001) Signal processing in magnetoencephalography. Methods 25:249-271.

Warren JE, Wise RJ, Warren JD (2005) Sounds do-able: auditory-motor transformations and the posterior temporal plane. Trends Neurosci 28:636-643.

Wildgruber D, Ackermann H, Grodd W (2001) Differential contributions of motor cortex, basal ganglia, and cerebellum to speech motor control: effects of syllable repetition rate evaluated by fMRI. Neuroimage 13:101-109.

Womelsdorf T, Schoffelen JM, Oostenveld R, Singer W, Desimone R, Engel AK, Fries P (2007) Modulation of neuronal interactions through neuronal synchronization. Science 316:1609-1612. 